\title{
Parameter Variation Analysis for Voltage Controlled Oscillators in Phase-Locked Loops
}

\author{
Igor Vytyaz, David C. Lee ${ }^{\dagger}$, Un-Ku Moon, and Kartikeya Mayaram \\ Oregon State University, Corvallis, Oregon, USA \\ $\dagger$ Berkeley Design Automation, Santa Clara, California, USA
}

\begin{abstract}
A new oscillator sensitivity analysis that predicts the impact of parameter variations of a VCO in a PLL is presented in this paper. Sensitivities of an oscillator's steady-state performance to design, process, or environmental parameter variations can be accurately and efficiently computed with the proposed analysis.
\end{abstract}

\section{INTRODUCTION}

Analysis techniques that can predict the impact of parameter variations on circuit performance are important for circuit design. They not only provide information about parameters that are critical in achieving the specifications, but also pave the way for design optimization. For an oscillator, an important block in communication circuits, the sensitivities of the periodic steady-state (PSS) solution and the perturbation projection vector (PPV) are needed to evaluate the impact of process and environmental parameter variations. Sensitivity analysis for free-running oscillators has been previously developed in [1].

However, in a phase-locked loop (PLL), the oscillation frequency of a voltage-controlled oscillator (VCO) is specified by the reference frequency and division settings. In a closed-loop configuration, VCO parameter variations in a VCO have no effect on the oscillation frequency as the PLL adjusts the control voltage to keep the frequency unchanged. More importantly, given a parameter variation, the PSS solution and the PPV of closed-loop and free-running oscillators vary in a different manner. Therefore, the sensitivity analysis for freerunning oscillators as described in [1] can not be used to analyze oscillators in a PLL, and an alternative formulation is needed.

In this paper, a sensitivity analysis that is applicable for oscillators with a fixed oscillation frequency (closed-loop oscillator) is presented. The new sensitivity analysis is based on the PSS analysis for oscillators with a specified oscillation frequency (PSS-SF) [2] and also can be used in oscillators where the oscillation frequency is a design specification, and the control voltage is not an appropriate frequency-tuning parameter [2]. Instead, the tank capacitor or the device size in a delay cell is used as a frequency tuning-parameter in $L C$ or ring oscillators, respectively. If a design parameter is changed, the frequency-tuning parameter must be changed accordingly, such that the oscillation frequency meets the design specification. The change in the frequency-tuning parameter, as well as the PSS solution and PPV can be predicted with the alternative sensitivity analysis presented in this paper.

The PSS-SF analysis for oscillators and PPV are reviewed in Section II. In Section III, continuous-time descriptions of an oscillator's PSS and PPV sensitivities are presented. Sensitivity of a ring VCO is analyzed in Section IV. It is shown how the oscillator sensitivity analysis can be used in applications such as predicting the impact of VCO parameter variations, and VCO macromodeling for fast PLL analysis. Finally, the paper is concluded in Section V.

\section{FUNDAMENTAL OSCILLATOR CHARACTERISTICS}

In this section, the periodic steady-state and the perturbation projection vector of oscillators are defined. A continuous-time description of these fundamental oscillator characteristics is presented.

\section{A. Periodic Steady State (PSS)}

Any nonlinear oscillator circuit can be modeled as a set of $m$ differential-algebraic equations (DAEs) given by

$$
\frac{d}{d t} q\left(x(t), \gamma_{f_{0}}\right)+f\left(x(t), \gamma_{f_{0}}\right)+b\left(\gamma_{f_{0}}\right)=0
$$

where

$$
\begin{aligned}
& t \in \mathbb{R}: \text { time, independent variable, } \\
& \gamma_{f_{0}} \in \mathbb{R}: \text { frequency-tuning parameter, } \\
& x: \mathbb{R} \rightarrow \mathbb{R}^{m}: \text { oscillator state variables, } \\
& q: \mathbb{R}^{m} \times \mathbb{R} \rightarrow \mathbb{R}^{m}: \text { contribution of reactive components, } \\
& f: \mathbb{R}^{m} \times \mathbb{R} \rightarrow \mathbb{R}^{m}: \text { contribution of resistive components, } \\
& b: \mathbb{R} \rightarrow \mathbb{R}^{m}: \text { independent sources. }
\end{aligned}
$$

The $T$-periodic solution $x(t)$ of DAEs in (1) is called the PSS solution if it satisfies $x(t)=x(t+T)$. This periodicity constraint can be expressed as

$$
x(0)=x(T)
$$

Notice that if $x(t)$ is a PSS solution, then $x(t+\Delta t), \forall \Delta t$ is also a valid PSS solution. A unique isolated solution can be selected by imposing a phase condition

$$
\varphi(x(0))=0, \quad \varphi: \mathbb{R}^{m} \rightarrow \mathbb{R}
$$

One possible phase condition is to let a component of $x(0)$ be a fixed value.

The oscillator PSS is uniquely defined by (1), (2), and (3), resulting in the continuous-time equations for the oscillator in the steady-state

$$
\left\{\begin{array}{l}
\frac{d}{d t} q\left(x(t), \gamma_{f_{0}}\right)+f\left(x(t), \gamma_{f_{0}}\right)+b\left(\gamma_{f_{0}}\right)=0 \\
x(0)=x(T) \\
\varphi(x(0))=0
\end{array}\right.
$$

This is a periodic boundary value problem (BVP), a special case of a two-point BVP [4]. Given the desired value of the oscillation period $T=T_{\text {target }}$, the PSS-SF analysis finds the PSS solution $x_{s}$ and the value of the frequency-tuning parameter $\gamma_{f_{0}}$ by solving (4) in the time domain by the finite difference, or shooting methods, as well as in the frequency domain by the harmonic balance method [2]. 


\section{B. Perturbation Projection Vector (PPV)}

The PPV $v_{1}(t), v_{1}: \mathbb{R} \rightarrow \mathbb{R}^{m}$ is a $T$-periodic vector that quantitatively describes how additive noise is converted by the oscillator into phase deviation [3].

Consider a system of $m$ linear DAEs in $x(t)$

$$
\frac{d}{d t}(C(t) x(t))+G(t) x(t)=0
$$

with $T$-periodic coefficients

$$
C(t) \equiv C\left(x_{s}(t), \gamma_{f_{0}}\right)=\left.\frac{\partial q\left(x, \gamma_{f_{0}}\right)}{\partial x}\right|_{x_{s}(t)}, C: \mathbb{R}^{m} \times \mathbb{R} \rightarrow \mathbb{R}^{m \times m}
$$

and

$$
G(t) \equiv G\left(x_{s}(t), \gamma_{f_{0}}\right)=\left.\frac{\partial f\left(x, \gamma_{f_{0}}\right)}{\partial x}\right|_{x_{s}(t)}, G: \mathbb{R}^{m} \times \mathbb{R} \rightarrow \mathbb{R}^{m \times m}
$$

known as the capacitance and conductance matrices, respectively. The system in (5) is obtained by differentiation of (1) with respect to $x$ at the PSS solution. The corresponding adjoint system of $m$ linear DAEs in $y(t)$ is given by

$$
C^{T}(t) \frac{d}{d t} y(t)-G^{T}(t) y(t)=0
$$

The only periodic basis for the solution to (6) is the PPV $v_{1}(t)$ [3]. A properly scaled PPV is selected by requiring that

$$
-v_{1}^{T}(t) C(t) \dot{x}_{s}(t)=1, \quad \forall t
$$

The oscillator PPV is defined by the system of linear DAEs in $y(t)$

$$
\left\{\begin{array}{l}
C^{T}(t) \frac{d}{d t} y(t)-G^{T}(t) y(t)=0 \\
y(0)=y(T) \\
-y^{T}(0) C(0) \dot{x}_{s}(0)=1
\end{array}\right.
$$

which ensures that the solution $v_{1}(t)$ satisfies the adjoint system, is periodic, and is properly scaled. The PPV is found by solving (8) directly in the time or frequency domains [3], or by reducing it to an initial value problem (IVP) [3].

\section{Sensitivity ANALYSiS FOR OSCILlators WITH A SPECIFIED FREQUENCY}

An oscillator's PSS and PPV can change in response to a change in the design, process, or environmental parameters of an oscillator. The value of a frequency-tuning parameter also changes to keep the specified oscillation frequency unchanged. In this section, the sensitivities of these fundamental oscillator characteristics to the parameter are defined. A continuous-time mathematical description of PSS and PPV sensitivities for oscillators with a specified frequency is derived. This is accomplished by differentiating the continuoustime oscillator equations presented in Section II with respect to the parameter.

Let $\gamma_{p} \in \mathbb{R}$ be an oscillator design parameter, such as MOSFET geometry parameters $W_{M}, L_{M}$, values of passive components $R$, $L, C$, process parameters, or environmental parameters, such as temperature, power supply voltage, etc. The oscillator component contributions $q, f$, their derivatives $C, G$, independent sources $b$, the frequency-tuning parameter $\gamma_{f_{0}}$, PSS and PPV waveforms $x_{s}, v_{1}$ are now functions of $\gamma_{p}$, and the specified period $T=T_{\text {target }}$ does not depend on $\gamma_{p}$.

The sensitivity of the frequency-tuning parameter $\gamma_{f_{0}}$ with respect to $\gamma_{p}$, evaluated at a specific value of $\gamma_{p}=\gamma_{p}^{*}$, is the rate at which $\gamma_{f_{0}}$ changes with respect to a small change $\Delta \gamma_{p}$ in $\gamma_{p}$ and is given by

$$
\left.\frac{d \gamma_{f_{0}}\left(\gamma_{p}\right)}{d \gamma_{p}}\right|_{\gamma_{p}^{*}}=\lim _{\Delta \gamma_{p} \rightarrow 0} \frac{\gamma_{f_{0}}\left(\gamma_{p}^{*}+\Delta \gamma_{p}\right)-\gamma_{f_{0}}\left(\gamma_{p}^{*}\right)}{\Delta \gamma_{p}}
$$

Consider a $T$-periodic waveform $s\left(t, \gamma_{p}\right)$, which can be the PSS waveform $x_{s}$, or the PPV waveform $v_{1}$. The sensitivity of this waveform with respect to $\gamma_{p}$ is given by

$$
\left.\frac{d s\left(t, \gamma_{p}\right)}{d \gamma_{p}}\right|_{\gamma_{p}^{*}}=\lim _{\Delta \gamma_{p} \rightarrow 0} \frac{\Delta_{t} s}{\Delta \gamma_{p}}, \quad \forall t
$$

with

$$
\Delta_{t} s=s\left(t, \gamma_{p}^{*}+\Delta \gamma_{p}\right)-s\left(t, \gamma_{p}^{*}\right)
$$

Thus, the PSS and PPV sensitivity analyses for oscillators with a specified frequency find the sensitivity of the frequency-tuning parameter $d \gamma_{f_{0}} / d \gamma_{p}$ as in (9), and $T$-periodic sensitivities of PSS and PPV waveforms $d x_{s} / d \gamma_{p}$ and $d v_{1} / d \gamma_{p}$ as in (10).

The sensitivity analysis for free-running oscillators [1] uses the normalized time $\tau$ as the independent variable. In the sensitivity analysis for oscillators with a specified frequency there is no need to use the normalized time, as the changes in $\gamma_{p}$ and $\gamma_{f_{0}}$ compensate for each other and the oscillation period $T$ remains unchanged.

\section{A. Periodic Steady-State Sensitivity Analysis}

The PSS sensitivity for oscillators with a specified frequency is obtained by a differentiation of the PSS-SF equations in (4) with respect to the parameter $\gamma_{p}$ at the steady-state $x_{s}$. Recall that not only $x$, and $\gamma_{f_{0}}$ but also $q, f$, and $b$ are functions of $\gamma_{p}$.

The contribution of the resistive circuit components to (4) depend on $\gamma_{p}$ directly, as well as indirectly through the PSS waveform $x_{s}$ and the frequency-tuning parameter $\gamma_{f_{0}}$ that are affected by $\gamma_{p}$. Therefore, the total derivative of $f$ with respect to $\gamma_{p}$ is composed of three terms

$$
\begin{aligned}
& \frac{d}{d \gamma_{p}}\left[f\left(x\left(t, \gamma_{p}\right), \gamma_{f_{0}}\left(\gamma_{p}\right), \gamma_{p}\right)\right] \\
& =\underbrace{\frac{\partial f}{\partial \gamma_{p}}}_{1}+\underbrace{G \cdot \frac{d x}{d \gamma_{p}}}_{2}+\underbrace{\frac{\partial f}{\partial \gamma_{f_{0}}} \cdot \frac{d \gamma_{f_{0}}}{d \gamma_{p}}}_{3}
\end{aligned}
$$

which have the following interpretations:

1. Direct effect of parameter $\gamma_{p}$ on $f$. Resistive circuit components that directly depend on $\gamma_{p}$, such as a resistor with resistance being the parameter $\gamma_{p} \equiv R$, contribute to this term.

2. Chain effect of parameter $\gamma_{p}$ on $f$ caused by a change in the PSS waveform $x_{s}$. Resistive circuit components, such as a resistor, or a MOSFET, contribute to this term.

3. Chain effect of parameter $\gamma_{p}$ on $f$ caused by a change in the frequency-tuning parameter $\gamma_{f_{0}}$. The change in $\gamma_{f_{0}}$ compensates for the change in the parameter $\gamma_{p}$ to keep the specified oscillation period unchanged.

The total derivative of the contribution of the reactive circuit components with respect to parameter $\gamma_{p}$ is expressed similar to (12).

Therefore, the PSS sensitivity equations for oscillators with a specified frequency are given by a system of linear DAEs that represent a BVP in $d x / d \gamma_{p}$ and $d \gamma_{f_{0}} / d \gamma_{p}$

$$
\left\{\begin{array}{l}
\frac{d}{d t}\left(C \frac{d x}{d \gamma_{p}}\right)+G \frac{d x}{d \gamma_{p}}+\frac{\partial}{\partial \gamma_{f_{0}}}\left[\frac{d q}{d t}+f+b\right] \frac{d \gamma_{f_{0}}}{d \gamma_{p}}=-R_{\mathrm{PSS}}(t) \\
\frac{d x\left(0, \gamma_{p}\right)}{d \gamma_{p}}-\frac{d x\left(T, \gamma_{p}\right)}{d \gamma_{p}}=0 \\
\left.\frac{\partial}{\partial x} \varphi(x)\right|_{x\left(0, \gamma_{p}\right)} \frac{d}{d \gamma_{p}} x\left(0, \gamma_{p}\right)=0
\end{array}\right.
$$


where

$$
R_{\mathrm{PSS}}(t)=\frac{\partial}{\partial \gamma_{p}}\left[\frac{d q}{d t}+f+b\right]
$$

is a periodic forcing term that depends on the choice of $\gamma_{p}$.

Computation of PSS sensitivity requires a PSS-SF analysis to be first performed to obtain the PSS waveforms $x_{s}$, and the value of the frequency-tuning parameter $\gamma_{f_{0}}$. Assuming that the underlying PSS-SF analysis in based on the Newton-Raphson method, the periodic matrix coefficients $C$, and $G$ in (13) are also available. The derivatives in (13) $\partial[\dot{q}+f+b] / \partial \gamma_{f_{0}}$ and (14) $\partial[\dot{q}+f+b] / \partial \gamma_{p}$ are obtained from device models at the steady-state $x_{s}$.

Once assembled, the continuous-time PSS sensitivity equations in (13) can be solved in the time domain by the finite-difference, or shooting methods, as well as in the frequency domain by the harmonic balance method for $d x_{s} / d \gamma_{p}$, and $d \gamma_{f_{0}} / d \gamma_{p}$.

In optimization problems, the gradient of an objective function $\nabla F_{o b j}$ with respect to a $P$-vector of design parameters $\left[\gamma_{1}, \ldots, \gamma_{P}\right]^{T}$ is often needed. In this case, (13) must be solved $P$ times with different forcing terms $R_{\text {PSS }}$ to compute PSS sensitivities with respect to all optimization parameters $\gamma_{p}, p=1, \ldots, P$. The periodic coefficients $C, G$, and $\partial[\dot{q}+f+b] / \partial \gamma_{f_{0}}$ are properties of the periodic steady-state, and do not depend on the choice of parameter $\gamma_{p}$.

The PSS sensitivity DAEs in (13) are linear, and therefore, easier and faster to solve than the nonlinear DAEs for the PSS-SF analysis in (4). Therefore, the proposed PSS sensitivity analysis for oscillators with a specified oscillation frequency is more efficient compared to finding a numerical approximation of PSS sensitivity, which requires an additional nonlinear PSS-SF analysis with a perturbed value of the parameter. With multiple parameters, this is a significant benefit of the proposed PSS sensitivity analysis.

\section{B. Perturbation Projection Vector Sensitivity Analysis}

The PPV sensitivity $d v_{1} / d \gamma_{p}$ is the solution of the linear system of DAEs in $d y / d \gamma_{p}$

$$
\left\{\begin{array}{l}
C^{T} \frac{d}{d t} \frac{d y}{d \gamma_{p}}-G^{T} \frac{d y}{d \gamma_{p}}=-R_{\mathrm{PPV}}(t) \\
\frac{d y\left(0, \gamma_{p}\right)}{d \gamma_{p}}-\frac{d y\left(T, \gamma_{p}\right)}{d \gamma_{p}}=0 \\
{\left[C \dot{x}_{s}\right]^{T} \frac{d y\left(0, \gamma_{p}\right)}{d \gamma_{p}}=-v_{1}^{T} \frac{d}{d \gamma_{p}}\left[C \dot{x}_{s}\right], \quad t=0}
\end{array}\right.
$$

with

$$
R_{\mathrm{PPV}}(t)=\frac{d C^{T}}{d \gamma_{p}} \frac{d v_{1}}{d t}-\frac{d G^{T}}{d \gamma_{p}} v_{1}
$$

that is obtained by differentiation of the PPV description in (8) with respect to $\gamma_{p}$ at $y=v_{1}$. Not only $y$ but also $C, G, x_{s}$, and $\gamma_{f_{0}}$ have to be treated as functions of $\gamma_{p}$ in order to obtain (15).

The total derivative of the capacitance matrix with respect to parameter $\gamma_{p}$ is expressed similar to (12)

$$
\frac{d}{d \gamma_{p}} C\left(x, \gamma_{f_{0}}, \gamma_{p}\right)=\frac{\partial C}{\partial \gamma_{p}}+\frac{\partial C}{\partial x} \cdot \frac{d x}{d \gamma_{p}}+\frac{\partial C}{\partial \gamma_{f_{0}}} \cdot \frac{d \gamma_{f_{0}}}{d \gamma_{p}}
$$

with

$$
\frac{\partial C}{\partial x} \cdot \frac{d x}{d \gamma_{p}} \equiv \sum_{j=1}^{m}\left(\frac{\partial C}{\partial x_{j}} \cdot \frac{d x_{j}}{d \gamma_{p}}\right)
$$

where $x_{j}$ is the $j$-th entry of $x$. The expression for the total derivative of the conductance matrix with respect to $\gamma_{p}$ is similar to (18). These derivatives are obtained from device models at the steady-state $x_{s}$.

Computation of the PPV sensitivity requires PSS (or PSS-SF) and PPV analyses to be first performed to obtain $v_{1}, C, G$, and $x_{s}\left(0, \gamma_{p}\right)$ in (15) and (16). The sensitivities $d \gamma_{f_{0}} / d \gamma_{p}$, and $d x_{s} / d \gamma_{p}$ required in (15) and (18) are obtained from the PSS-SF sensitivity analysis described in Section III-A.
Once the BVP in (15) is assembled, the PPV sensitivity $d v_{1} / d \gamma_{p}$ is obtained by solving it in the time or frequency domains, or by reducing it to an IVP.

\section{EXAMPLES AND RESUltS}

We have implemented the PSS and PPV sensitivity analyses for closed-loop oscillators in our Matlab-based circuit simulator, and in Berkeley Design Automation's RF FastSPICE.

In this section the sensitivity of a ring VCO is analyzed using a time-domain numerical method. It is shown that the PSS and PPV sensitivities can provide valuable information for predicting the impact of VCO parameter variations on PLL performance. In addition, a VCO phase macromodel can be generated that accurately accounts for these variations.

The oscillator under consideration is a three-stage ring VCO in Figure 1 consisting of three identical inverters, and a control device $M_{c}$.

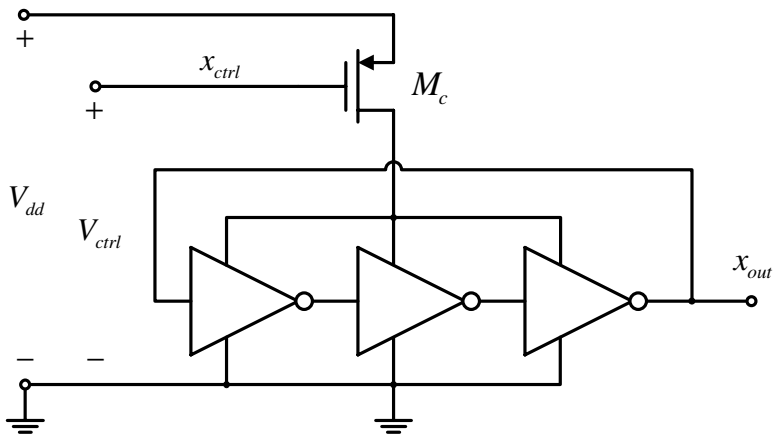

Fig. 1. Schematic of a three-stage ring VCO.

Consider a simple PLL with a divider of $N=1$. In the PLL, the control voltage $V_{c t r l}$ settles to $3.61 \mathrm{~V}$, such that the oscillation frequency of the VCO $f_{0}=1 / T$ is the same as the reference frequency $f_{r e f}=1 \mathrm{GHz}$, i.e., the PLL is in lock.

Next, we analyze how the PLL performance is affected by a $20 \%$ variation in the VCO parameter $\gamma_{p} \equiv W_{c}$, the width of the control device. The designed value of the control device width is $W_{c}^{*}=18 \mu \mathrm{m}$, and the variation is $\Delta W_{c}=3.6 \mu \mathrm{m}$.

The VCO sensitivity analysis with the control voltage as a frequency-tuning parameter $\gamma_{f_{0}} \equiv V_{c t r l}$ is used to obtain the sensitivity of the control voltage

$$
\frac{d V_{c t r l}}{d W_{c}}=\frac{d \gamma_{f_{0}}}{d \gamma_{p}}=27.4 \frac{\mathrm{mV}}{\mu \mathrm{m}}
$$

This sensitivity allows us to predict the value of the control voltage in the PLL with parameter variation as a linear approximation

$$
\begin{aligned}
& V_{\text {ctrl }}\left(W_{c}^{*}\right)+\Delta W_{c} \frac{d V_{c t r l}}{d W_{c}} \\
& =3.61 \mathrm{~V}+3.6 \mu \mathrm{m} \cdot 27.4 \frac{\mathrm{mV}}{\mu \mathrm{m}}=3.71 \mathrm{~V}
\end{aligned}
$$

This value is close to actual value of the control voltage $V_{c t r l}\left(W_{c}^{*}+\Delta W_{c}\right)=3.70 \mathrm{~V}$, found from a PSS-SF analysis, in which the VCO with parameter variation oscillates at $1 \mathrm{GHz}$.

Another important characteristic of the VCO is the PPV. The PPV $v_{1 V_{d d}}$ corresponding to the $V_{d d}$ equation is a measure of how much noise from the power supply is projected into the phase deviation. Therefore, a VCO design with the smallest PPV magnitude has the best power supply rejection. Figure 2 shows the PPV of the original VCO $v_{1 V_{d d}}\left(W_{c}^{*}\right)$ and the predicted PPV of the VCO with parameter 
variation $v_{1 V_{d d}}\left(W_{c}^{*}\right)+\Delta W_{c} \cdot d v_{1 V_{d d}} / d W_{c}$, obtained with the use of the PPV sensitivity analysis. It is seen that the predicted PPV accu-

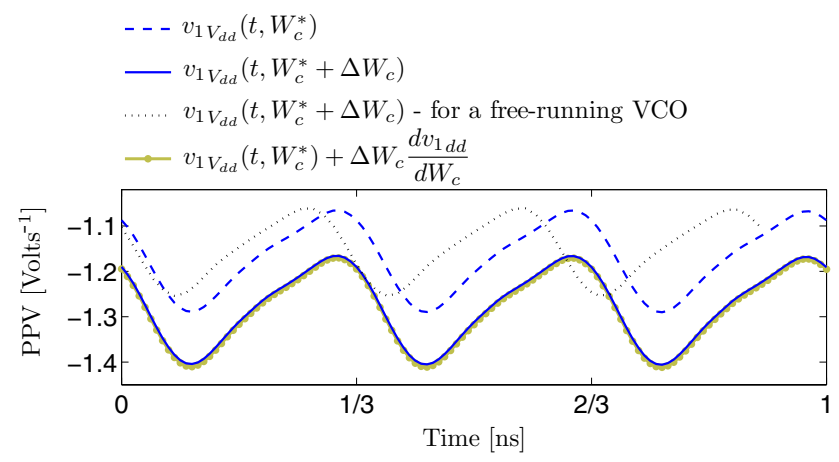

Fig. 2. The PPV for the $V_{d d}$ equation of the original VCO, and the VCO in the presence of parameter variation $\Delta W_{c}$. The PPV of the VCO with parameter variation is well-matched by the PPV predicted with the use of the sensitivity analysis for the closed-loop VCO. Given a parameter variation, the PPV of closed-loop and free-running VCOs change in a different manner.

rately matches the actual PPV $v_{1 V_{d d}}\left(W_{c}^{*}+\Delta W_{c}\right)$ of a VCO with parameter variation, thereby eliminating the need for an additional PPV analysis. Note that the variation $\Delta W_{c}$ causes the PPV $v_{1 V_{d d}}$ to increase in magnitude. Therefore, the $\mathrm{VCO}$, and, consequently, the PLL are more sensitive to the power supply noise.

In a free-running oscillator parameter variation affects the oscillation period rather than the frequency-tuning parameter. Consequently, as shown in Figure 2, the PPV of closed-loop and free-running VCOs change in different ways. This shows that the VCO with a specified frequency cannot be analyzed with the method presented in [1].

PPV-based VCO phase macromodels can be used to speed-up the transient simulation of a PLL by two orders of magnitude [5]. The sensitivity analysis can be used to generate a VCO phase macromodel that captures the effect of parameter variations. This macromodel eliminates the need for additional PSS-SF and PPV analyses for each set of parameter values in a Monte-Carlo analysis. The macromodel computes the phase deviation based on the predicted PPV. During a transient simulation of the PLL with the VCO macromodel, the difference between the voltage at the control node $x_{c t r l}(t)$, and the predicted control voltage is treated as a perturbation

$$
b_{n V_{c t r l}}(t)=x_{c t r l}(t)-\left[V_{c t r l}\left(W_{c}^{*}\right)+\Delta W_{c} \frac{d V_{c t r l}}{d W_{c}}\right]
$$

The results from a transient analysis of a PLL with the original transistor-level VCO (without any parameter variation), the transistorlevel VCO, and the macromodel of a $\mathrm{VCO}$ in the presence of parameter variation $\Delta W_{c}$ and power supply noise $b_{n V_{d d}}(t)$ are shown in Figure 3. The control and output voltages of the PLL are shown in Figures 3(b) and (c), respectively. It is seen that the VCO macromodel based on the proposed sensitivity analysis allows us to perform a fast transient simulation of a PLL in the presence of parameter variations without a significant loss in accuracy.

\section{CONCLUSION}

We have presented a continuous-time formulation of sensitivity analysis for oscillators with a specified frequency. The new sensitivity formulation enables the sensitivity calculation for oscillators in a PLL and those with a specified oscillation frequency. The new analysis is a useful tool for predicting the impact of parameter variations of a VCO in a PLL, macromodeling, and design optimization.

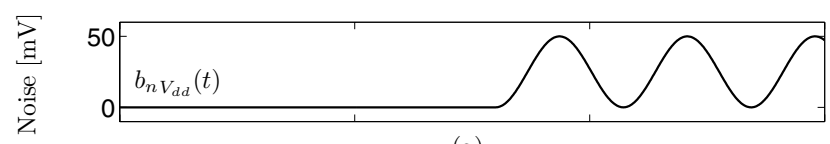

(a)
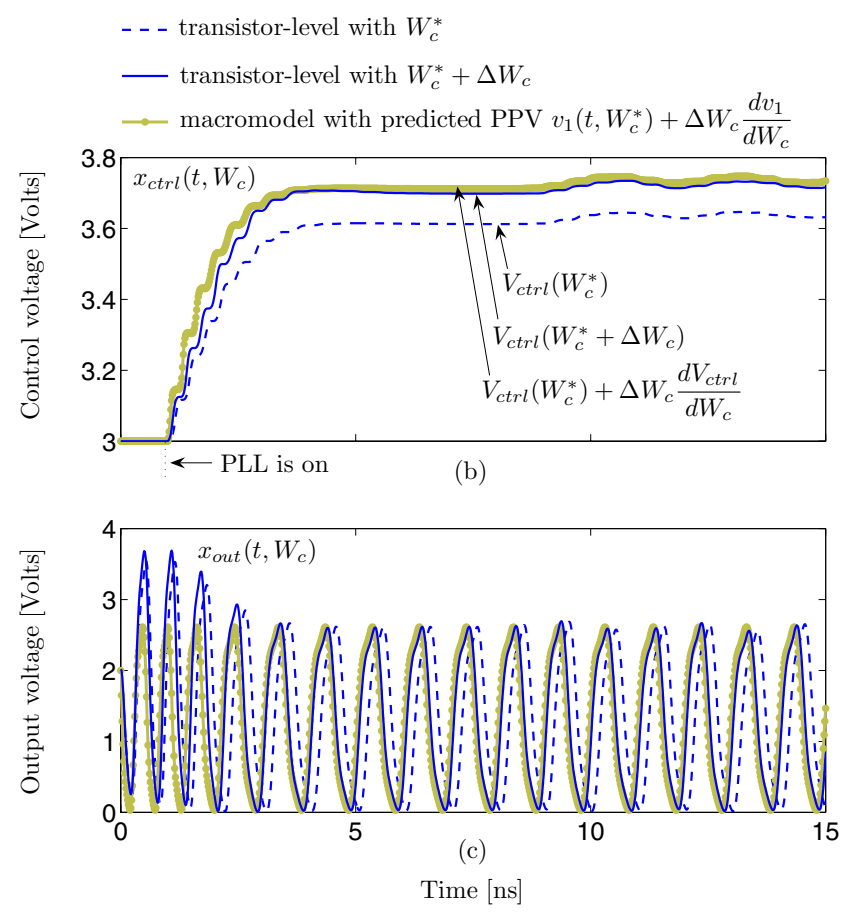

Fig. 3. (a) Power supply voltage noise, (b) PLL control voltage, and (c) PLL output voltage of the original PLL, and the PLL in the presence of parameter variation $\Delta W_{c}$. The transient response of the PLL with parameter variation from transistor-level simulation is in good agreement with the simulation of the VCO macromodel based on the VCO sensitivity analysis.

\section{ACKNOWLEDGMENT}

This research is supported in part by SRC under contract 2005-HJ-1326.

\section{REFERENCES}

[1] I. Vytyaz, D. C. Lee, P. Hanumolu, U. Moon, and K. Mayaram, "Sensitivity analysis for oscillators," ICCAD 2007, pp. 458-463, Nov 2007.

[2] I. Vytyaz, D. C. Lee, S. Lu, A. Mehrotra, Un-Ku Moon, and K. Mayaram, "Parameter finding methods for oscillators with a specified oscillation frequency," DAC 2007, pp. 424-429, June 2007.

[3] A. Demir, "Floquet theory and non-linear perturbation analysis for oscillators with differential-algebraic equations," International Journal of Circuit Theory and Applications, vol. 28, pp. 163-185, Mar-Apr. 2000.

[4] U. M. Ascher, R. M. M. Mattheij, and R. D. Russell, Numerical Solution of Boundary Value Problems for Ordinary Differential Equations, SIAM, 1995.

[5] X. Lai, Y. Wan, J. Roychowdhury, "Fast PLL simulation using nonlinear VCO macromodels for accurate prediction of jitter and cycle-slipping due to loop non-idealities and supply noise," ASPDAC 2005, pp. 459-464, Jan. 2005. 